\title{
Analysis of focal information of individuals: Gaming Approach to C2C market
}

\author{
Hitoshi Yamamoto ${ }^{1}$, Kazunari Ishida ${ }^{2}$ and Toshizumi Ohta ${ }^{3}$ \\ ${ }^{1}$ Faculty of Business Administration, Rissho University, Shinagawa-ku, Tokyo 141-8602, \\ Japan \\ hitoshi@ris.ac.jp \\ ${ }^{2}$ Faculty of Policy Studies, University of Shimane, 1-236 Tangi, Hachioji City, Tokyo 192- \\ 8577, Japan. \\ k-ishida@u-shimane.ac.jp
3 The Graduate School of Information Systems, University of Electro-Communications, \\ Choufu-shi, Tokyo 182-8585, Japan \\ ohta@is.uec.ac.jp
}

\begin{abstract}
To analyze the effect of reputation management systems for promoting cooperative behavior in a $\mathrm{C} 2 \mathrm{C}$ market, we developed a virtual $\mathrm{C} 2 \mathrm{C}$ market system and experimented with participants to analyze transaction and information behaviors. According to the result of our experiment, we found that over $80 \%$ of participants behaved cooperatively. However, some participants accumulated high reputation in the early round of the experiment, and then exploited cooperative participants with the high reputation and defective action. The result indicates existence of vulnerability of reputation management system. Based on analysis of information behavior, we also found that cooperative participants often referred the number of defects and duration of ID unchanged. The result indicates cooperative participants prefer risk adverse to choose trustful others to make deal.
\end{abstract}

\section{Introduction}

The recent growth of $\mathrm{C2C}$ (consumer-to-consumer) market is one of the remarkable phenomena of the Internet, a medium which reduces the limitations of our lives in terms of distance, time, and opportunity. We can sell or buy virtually any goods to others on the Internet, something never possible before. eBay and Yahoo! Japan Auction are two successful examples of such marketplaces.

However, market growth also results in an increase of risk on transactions, namely the failure of buyers to pay for goods and the failure of sellers to send goods. In an online market, participants can easily change identities by changing handles, or onscreen user IDs, and enter or exit the market. As a result, they may take advantage of the opportunity to accept goods without payment or to accept payment without sending goods, actions which we define as defective behavior.

To discourage defective behavior, we need management systems to promote cooperative behavior among participants on an online $\mathrm{C} 2 \mathrm{C}$ market. A reputation 
management system (RMS) is one effective means employed by many online marketplaces such as eBay and Yahoo! Japan Auction. An RMS provides a mechanism for participants to evaluate each other and to share their evaluations.

Several studies have revealed that an RMS allows participants to behave cooperatively as they attempt to maximize their own profit (Kollock, 1999) (Yamamoto et. al. 2004). Online C2C marketplaces are commonly employing their own systems (Dellarocas, 2003). A typical RMS calculates a reputation score for each participant by summing and averaging ratings given by those who have made transactions with the participant. An RMS also provides other information such as individual comments and transaction history.

One question concerning reputation management systems is exactly which information is effective for selecting trustworthy transaction partners. Does the effectiveness of information depend on the role of the participant as a buyer or seller, or as a cooperative or defective participant? To find the answers, we designed an experiment using a virtual online $\mathrm{C} 2 \mathrm{C}$ market in which we observed transaction behavior in terms of cooperative and defective behavior. To determine which information is most often referenced by each type of participant, we also observed information behavior, i.e. frequently referred information. Based on our observations, we can propose an RMS design that effectively promotes cooperation.

\section{Reputation Management Systems and Evolution of Cooperative Behavior}

There are three general approaches to analyzing the effectiveness of a reputation management system in an online C2C market: case studies, computer simulations, and laboratory experiments.

In one example of the first approach, a case study of eBay transactions by McDonald (2002) reported that buyers with a high reputation score can sell their goods at high prices. Resnick and Zeckhouser (2001) reported that rate of positive evaluation was over $99 \%$ in all mutual evaluations on eBay.

Using the second approach of computer simulations, online C2C transactions can be modeled in terms of game theory, particularly iterative prisoners' dilemma (PD). Yamamoto et. al. (2004) analyzed C2C transactions with a computer simulation model and revealed that an RMS emphasizing positive feedback is effective in promoting cooperative behavior in an online market, whereas an RMS emphasizing negative feedback is effective for offline transactions.

In the third approach, a great deal of research based on laboratory experiments has analyzed cooperative behavior in reputation management systems also in terms of PD. Ruth and York (2004) investigated how the presentation of performance information affects stakeholders' attitudes towards firms that seek to enhance their reputation. The results indicate that consistency between source and information type determines the impact on degree of attitude change. Rice (2004) investigates PD in terms of existence and uncertainty of feedback information. The results indicate that social welfare and efficiencies of trade increase where feedback is allowed. Bolton et. al. (2004) compares trading in a market with feedback to a market without feedback. The 
results indicate that feedback mechanisms induce a substantial improvement in transaction efficiency.

However, it should be noted that these studies have not discussed what kind of feedback information is important in the decision-making process of selecting buyers or sellers, and the identification of such information is necessary in order to design an effective RMS. To identify these decision-making factors in our own experiment, we designed a virtual online $\mathrm{C} 2 \mathrm{C}$ market in which we observed transaction and information behavior in terms of cooperative or defective behavior, as well as what type of feedback information is more important to each type of participant.

\section{Modeling C2C Online Transactions}

In order to design an experimental model of a C2C online marketplace, we discuss transactions within the framework of prisoner's dilemma, in which the players are represented by buyers and sellers.

\subsection{Prisoners’ Dilemma}

A player who participates in a $\mathrm{C} 2 \mathrm{C}$ online transaction always has an incentive to cheat others (i.e., to defect) due to anonymity and the ease of entry and exit from transactions. On one hand, a buyer may accept goods from a seller without payment. On the other hand, a seller may accept payment from a buyer without sending goods. The situation in $\mathrm{C} 2 \mathrm{C}$ online transactions is representative of prisoner's dilemma. We can consider these strategies within a payoff matrix, as shown in Table $1(\mathrm{~T}>\mathrm{R}>\mathrm{P}>\mathrm{S})$.

Table 1: Payoff matrix for prisoner's dilemma

\begin{tabular}{|c|c|c|c|}
\hline & \multicolumn{3}{|c|}{ Action of player- 2} \\
\hline & & $\mathrm{C}$ & $\mathrm{D}$ \\
\hline & $\mathrm{C}$ & $\mathrm{R}, \mathrm{R}$ & S, T \\
\hline & $\mathrm{D}$ & T, S & $\mathrm{P}, \mathrm{P}_{2}$ \\
\hline
\end{tabular}

In the prisoner's dilemma of a C2C online transaction, a seller can take two possible actions: cooperation, i.e., sending goods in exchange for payment, and defection, accepting payment without sending goods. Likewise, a buyer can also cooperate or defect, i.e. pay for goods or accept goods without payment.

\subsection{Formulation of Transactions}

Each participant in our experiment was assigned a role of either buyer or seller. We denoted the item price, production cost, and utility of the item for each buyer by P, C, and $\mathrm{V}$, respectively. We assume profitable conditions, i.e. $\mathrm{V}>\mathrm{P}>\mathrm{C}>0$.

Based on the notations, we can define a payoff for a seller (T, R, P, S) as (P, P-C, 0 , $-\mathrm{C}$ ), where $\mathrm{T}, \mathrm{R}, \mathrm{P}$, and $\mathrm{S}$ denote gain or loss in four possible cases of PD: defect against a cooperating opponent, mutual cooperation, mutual defect, and cooperation 
with a defecting opponent, respectively. In the case of defect against a cooperating opponent, a seller accepts payment from a buyer but does not ship the item to the buyer. In this case, the seller gains $\mathrm{T}(=\mathrm{P})$ because the seller gains payment $(\mathrm{P})$ without loss of production cost (C). In the case of mutual cooperation, a seller sends the item to a buyer and accepts payment from the buyer. In this case, the seller gains $\mathrm{R}(=\mathrm{P}-\mathrm{C})$, because the seller gains payment $(\mathrm{P})$ and loses the production cost $(\mathrm{C})$ of the item. In the case of mutual defect, the seller does not send the item to the buyer, and the buyer does not send payment to the seller. In this case, the seller does not gain or lose anything $(\mathrm{P}=0)$. In the case of cooperation with a defecting opponent, the seller sends the item to the buyer, but the buyer does not send payment. In this case, the seller loses $S(=-C)$ because the seller pays the production cost $(C)$ of the item without gaining payment $(\mathrm{P})$ from the buyer.

We can likewise define a payoff for a buyer (T, R, P, S) as (V, V-P, 0, -P). In the case of defect with a cooperating opponent, the buyer accepts the item from the seller but does not send payment to the seller. In this case, the buyer gains $\mathrm{T}(=\mathrm{V})$ because the buyer enjoys utility (V) without payment to compensate for the seller's production cost. In case of mutual cooperation, the buyer sends payment to the seller and the buyer receives the item from the seller. In this case, the buyer gains $\mathrm{R}(=\mathrm{V}-\mathrm{P})$ because the buyer enjoys the utility of the item (V) after paying the price of the item. In the case of mutual defect, the buyer does not send payment to the seller, and the seller does not send the item. In this case, the buyer does not gain or lose anything $(\mathrm{P}=0)$. In the case of cooperation with a defecting opponent, the buyer sends payment to the seller, but the seller does not send the item to the buyer. In this case, the buyer loses $\mathrm{S}$ $(=-\mathrm{P})$ because the buyer pays the price of the item $(\mathrm{P})$ without receiving the item from the seller.

In our experiment, we assume that the higher the price of an item, the higher its utility to the buyer, accounting for a difference in utility between a commodity and a luxury item (e.g., daily food vs. jewelry). Based on such an assumption, we define a relation between $\mathrm{V}$ and $\mathrm{P}$ as $V=\alpha \mathrm{P}$, where $\alpha$ is called the utility coefficient, and buyable condition is $1<\alpha$. We also assume a relationship between the price and cost of an item, i.e. the higher the price of an item, the higher the production cost due to materials or technology. Based on this assumption, we define a relation between $\mathrm{C}$ and $\mathrm{P}$ as $C=\beta \mathrm{P}$, where $\beta$ is called the cost coefficient. Profitable condition is then $0<\beta<1$. The gains and losses of the buyer and the seller in all cases are summarized in Table 2.

Table 2: Payoff matrix in experiment in online C2C transactions

\begin{tabular}{|c|c|c|}
\hline & Buyer Cooperates & Buyer defects \\
\hline Seller & Buyer's gain: $(\alpha-1) \mathrm{P}$ & Buyer's gain: $\alpha$ P \\
cooperates & Seller's gain: $(1-\beta) \mathrm{P}$ & Seller's loss: $-\beta \mathrm{P}$ \\
\hline Seller & Buyer's loss: $-\mathrm{P}$ & Buyer's gain: 0 \\
defects & Seller's gain: $\mathrm{P}$ & Seller's gain: 0 \\
\hline
\end{tabular}




\section{Experiment Overview}

In this section, we explain how the virtual transaction system for our experiment was developed. We assigned all participants to one of two groups, sellers or buyers, and each participant participated in several transactions.

In our experiment, the system not only provided each participant with an initial handle, or onscreen user ID, but also allowed other handles if the participant wished to change it. It should be noted that a handle is simply a means of identification in an online market, and not a participant's real name.

On the system, participants took actions, e.g. bid and award, synchronously. Although in real online C2C markets buyers and sellers take actions asynchronously, we did not design the experiment this way in order to simplify the transaction process for ease of analysis. Before we can analyze an asynchronous situation, we must first analyze the basic mechanisms in a synchronous situation.

The system permitted a buyer to make multiple bids to sellers and a seller to make multiple awards to bidders.

In the last phase of a period, each participant was given the opportunity to change handles. If the participant decided to do so, the system provided a new handle randomly selected from the database.

Each participant was allowed to choose between cooperation or defect in a transaction. Cooperation meant sending payment to a seller or sending an item to a buyer. Defect meant either no payment or no shipment.

Each participant was able to view sorted lists of other participants' feedback information in terms of transaction history, item prices, number of cooperative transactions, the number of defective transactions, reputation score, and the total number of transactions. The transaction history displayed not only the date/time of the transaction but the handles of both buyer and seller and their respective actions. The reputation score was calculated by subtracting the number of defective actions from those of cooperative actions..

\subsection{Transaction Process}

In our experiment, the transaction process was composed of four steps: (a) sellers deciding item prices, (b) buyers bidding on items, (c) sellers awarding items to bidders, and (d) both buyers and sellers checking the results of transactions and deciding whether to change their handles. An administrator managed the transaction process synchronously to ensure that all participants made decisions in each step (Fig. $1)$.

In the first step, the item pricing phase [(a) of Fig. 1], each seller decided on the price at which to sell the item within a predefined range, e.g., between 100 and 800 .

We assumed that a seller could reap benefit at a certain constant rate in relation to the item price, meaning that the difference between price and production cost for a luxury item is greater than that for a commodity item.

Hence, a seller could reap greater benefit from selling a high-priced item than a low-priced item in a successful transaction. At the same time, however, the seller also faced a high risk in losing high-priced items if the transaction failed. In other words, 
the transaction was high risk and high return when the seller decided to sell a highpriced item, and the transaction was low risk and low return when the seller decided to sell a low-priced item.

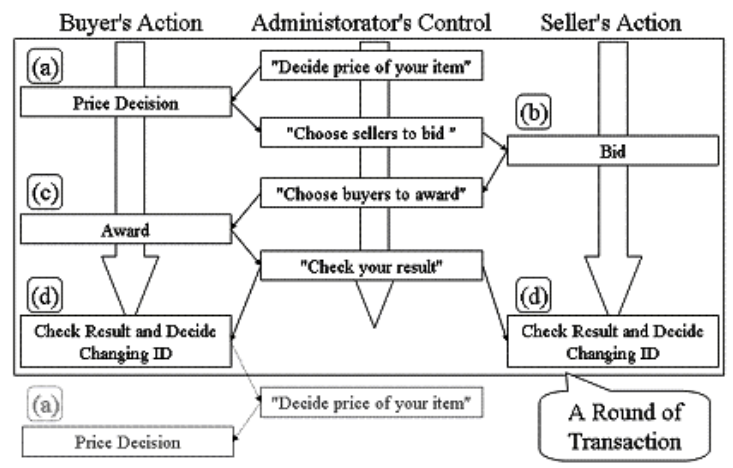

Fig. 1. Overview of transaction steps

In the second step, the bidding phase [(b) of Fig. 1], each buyer viewed feedback information for all sellers, then selected good sellers, and decided whether to make the payment. The action was applied to all sellers selected by the buyer in each round.

In the third step, the awarding of items [(c) of Fig. 1], each seller viewed feedback information for all bidders, then selected good buyers, and decided whether to send the item. The action was also applied to all buyers selected by the seller.

At this stage, all pairs of buyer and seller who have reached a deal agreement can gain or lose points based on the payoff matrix defined in 3.2 according to their actions and the price of the item. The system records their actions in their transaction histories for future reference.

In the last step [(d) of Fig. 1], all participants checked the transaction results, then decided whether to change handles, allowing them the opportunity to avoid any negative transaction history.

\subsection{Transaction System Overvie}

For our experiment, we developed a web-based, client and server online transaction system using an Apache web server and a MySQL database server with a PHP front end, similar to the systems of eBay and Yahoo! Japan Auction so that participants could use ours in the same way. Moreover, the technical requirements for using and administrating such a setup are simply computers equipped with web browsers and Internet access.We carried out the experiment and observed transaction and information behavior 


\section{Experiment Results}

We carried out the experiment and observed transaction and information behavior of its participants using the system explained in Section 4. The participants were 37 students at a university in Tokyo. They were divided into two groups consisting of 18 buyers and 19 sellers.

We performed 3 trial rounds so that participants could learn the system before the experiment itself, which consisted of 10 rounds. We did not inform the participants of the number of rounds in order to avoid its potential effect on decision-making. We provided rewards to the 5 highest-scoring participants as an incentive.

We defined two types of behaviors among participants: transaction behavior and information behavior. The former refers to cooperative or defective behavior. The latter refers to what information a participant references during decision-making.

\subsection{Analysis of Transaction Behavior}

Trajectories in Fig. 2 illustrate changes of the numbers of cooperative and defective actions. The figure illustrates an increase in cooperative action along with passage of time, as suggested by the high linear regression coefficient ( $\hat{\beta}=9.85$ ).

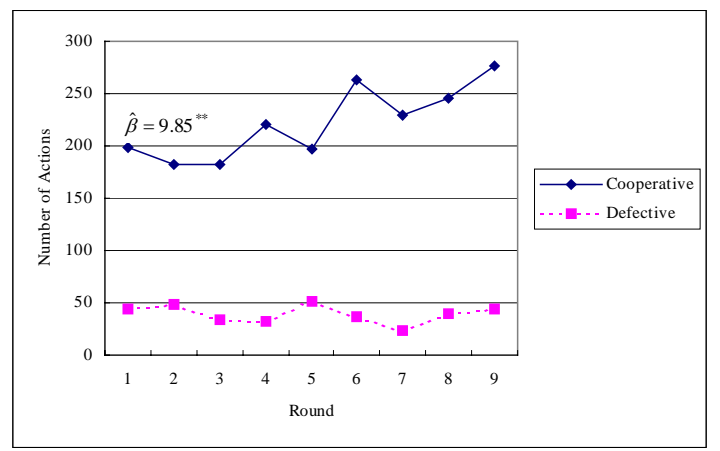

Fig. 2. Trajectories of cooperative and defective behavior

Fig. 3 illustrates the distribution of participants according to total profit and rate of cooperative action. We can distinguish three groups, which we will refer to as A, B, and $\mathrm{C}$, in the figure based on hierarchical clustering analysis (Fig. 4). In group A, which includes 32 of 37 participants, the higher the rate of cooperative action, the higher the profit each participant made. This tendency is further supported by the result of t-test for two sub groups within group A, which divided into those who were always cooperative and those who were sometimes defective (Tab. 3).

The three participants in group B obtained varying degrees of high profit with cooperative and defective actions. The final two participants in group $\mathrm{C}$ remained at low profit due to their consistently defective actions. 
Hitoshi Yamamoto,Kazunari Ishida, Toshizumi Ohta

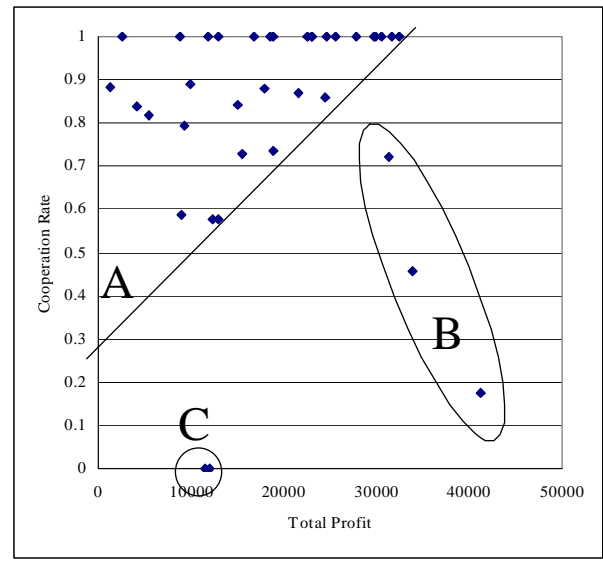

Fig. 3. Distribution of participants according to profit and cooperative behavior

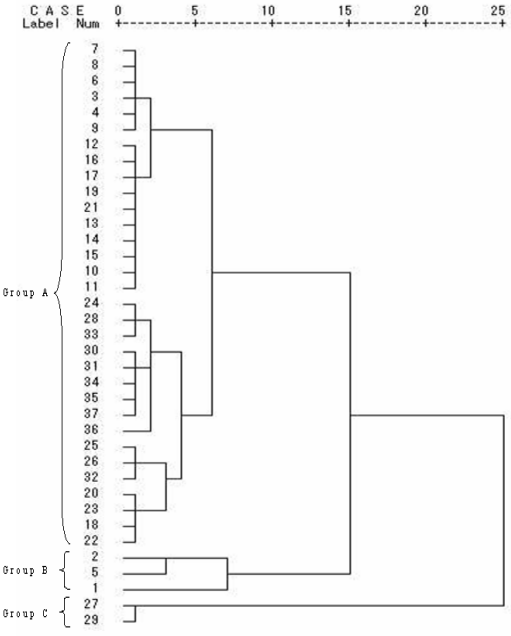

Fig. 4. Hierarchical clustering analysis of profit and ratio of cooperative action

Table 3: Result of t-test for two sub groups in group A in terms of profit Payoff matrix in experiment in online $\mathrm{C} 2 \mathrm{C}$ transactions

\begin{tabular}{ll}
\hline & Profit \\
\hline Always cooperative (18 members) & 21698.6 \\
\hline Sometime defective (14 members) & 12675.0 \\
\hline t-value & $3.245^{* *}$ \\
\hline$p$-value & 0.003 \\
\hline${ }^{* *}<.01$ &
\end{tabular}

Table 4: Cumulative number of information behaviors

\begin{tabular}{llllllc}
\hline & Reputation & C. & Defect & $\begin{array}{l}\text { ID } \\
\text { Duration }\end{array}$ & $\begin{array}{l}\text { Detailed } \\
\text { history }\end{array}$ & $\begin{array}{l}\text { Number of } \\
\text { Transaction }\end{array}$ \\
\hline C. & 2.94 & 3.85 & 4.76 & 3.92 & 1.18 & 1.24 \\
\hline $\begin{array}{l}\text { Defect } \\
\text { sometime }\end{array}$ & 3.89 & 5.81 & 2.89 & 1.06 & 2.03 & 1.54 \\
\hline t value & 0.55 & 1.33 & $1.94^{+}$ & $3.59^{* * *}$ & 0.60 & 0.34 \\
\hline p value & 0.58 & 0.18 & 0.05 & 0.00 & 0.55 & 0.73 \\
\hline${ }^{+} p<.10^{*} p<.05^{* *} p<.01^{* * *} p<.001$ & ( C. = Cooperation) & &
\end{tabular}




\subsection{Analysis of Information Behavior}

During this experiment, the total number of references to information by all participants was 739. The number of references to the price of an item was only 5 . The low number may suggest that finding cooperative participants is more important than finding participants who want to buy or sell high price items.

Fig 5 shows each cumulative total of information behaviors according to the criteria explained in Section 4. To analyze differences in information behavior between cooperative and defective participants, we categorized participants into two categories: always cooperative (participants who never defected against others) and sometimes defective (participants who took one or more defective actions against others) ${ }^{1}$. We normalized the number of information behaviors because of the difference among the numbers of references by the participants.

In addition, we ignored the information behaviors concerning price, which totaled to 11 , because of the relatively low number.

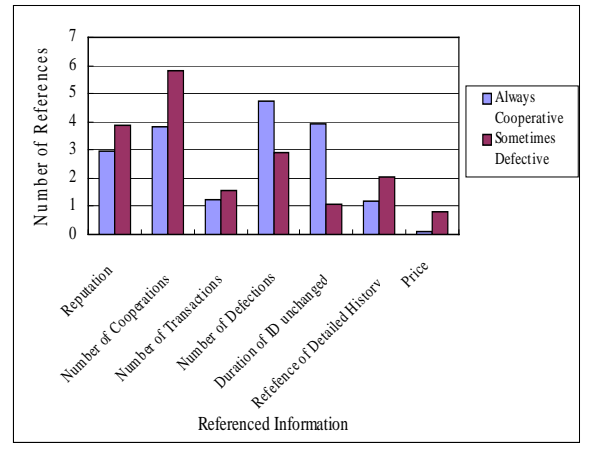

Fig. 5. Cumulative total of information behaviors

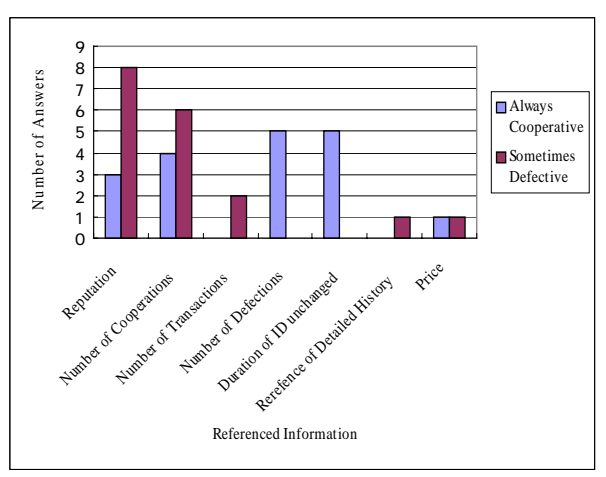

Fig. 6. C Information focus

According to Fig. 5 and Tab. 4, cooperative participants focused on the number of defective actions taken and the duration that a user ID remained unchanged. Hence these are the most effective factors to discriminate between cooperative and defective participants.

Fig. 6 summarizes the number of answers for a question in interviews to the participants after the experiment ${ }^{3)}$. The question is "What type of information is the most important to make decision to select the others to make deal?” The result in fig. 5 shows that cooperative participants focus on the number of defect and duration of ID unchanged, even though sometime defective participants completely ignore them.

We also classified answers to the question of which strategy is the most effective for maximizing profit into three categories in table 5 . 
Hitoshi Yamamoto,Kazunari Ishida, Toshizumi Ohta

Table 5: Effective strategy for maximizing profit

\begin{tabular}{|l|r|}
\hline \multicolumn{1}{|c|}{ Strategy } & Num \\
\hline Always cooperative & 18 \\
\hline $\begin{array}{l}\text { Early round cooperation, high reputation } \\
\text { obtained, and then defect in big deals }\end{array}$ & 14 \\
\hline Always defect, while changing ID & 2 \\
\hline Other & 2 \\
\hline
\end{tabular}

\section{Discussion}

As shown in Fig. 2 and 3, a reputation management system (RMS) promotes cooperative behaviors in the market because each participant discriminates between cooperative and defective others.

\subsection{Transaction Behavior and Fundamental Flaws of RMS}

Fig. 3 illustrates two insightful facts of a reputation management system. On one hand, group A, whose shape is a rectangle, depicts that choosing cooperative behavior gives a participant the chance to increase profit. This supports that RMS can promote cooperative behaviors by participants in the market.

However, on the other hand, in our interview after the experiment, the three participants in group B said that they had accumulated high reputation in the early rounds of the experiment, then exploited cooperative participants with their high reputation scores and defective action. The result indicates the vulnerability of RMS.

Such crimes have often occurred in recent years ${ }^{4)}$. A group may send items to buyers at first and respond to their claims quickly, to accumulate good reputation scores. When the group had become trusted by potential buyers, it exhibited large number of expensive items at reduced prices. After it received payments from all its buyers, the group disappeared from the online market.

The real-life examples of actual crime and our own experiment results emphasize that it is difficult to protect cooperative participants completely from fraud by malicious participants by employing an RMS by itself. Therefore, to compensate for the fundamental flaw of the ordinary RMS, it should be supplemented with another system, e.g. a legal system.

\subsection{Information Behavior and Redesigning RMS}

Based on our analysis of information behavior, we found significant differences between purely cooperative participants who never defected against other participants, and the others. All the purely cooperative participants often referenced 
two types of information: the duration that the handle remained unchanged and the number of defective actions. The tendency indicates that cooperative participants prefer transactions with other risk-adverse participants.

Based on our conclusions concerning relation between transaction and information behaviors, we can formulate a strategy to detect malicious sellers. Based on transaction behavior, the detectable signs are high reputation scores and switching from a low volume of inexpensive items to a high volume of expensive that. In the post-experiment interview, participants in group B sold inexpensive items to accumulate high reputation scores in the early rounds of transactions, then defected against buyers in transactions involving expensive items. Based on information behavior, the detectable sign is that the behavior is not risk-adverse, which means that the player could defect against others as shown in Fig. 3.

Employing such signs to detect malicious sellers, online marketplaces can monitor high-risk sellers to protect buyers, and we can reduce dependency on legal systems for secure online $\mathrm{C} 2 \mathrm{C}$ transactions. We propose a combination of the two signs to detect defective participants as one of the principles in redesigning RMS.

To make an RMS effective in protecting cooperative participants, extensive historical data must be archived to calculate reputation scores. Moreover, a participant who has not been selected by the others to make transactions might not be trustworthy, even though he or she has used the same handle for a long time. Hence, we suggest that the number of past transactions is as important as the number of defective actions and duration that the handle has remained unchanged. We also propose the combination of these three types of information as another principle in redesigning RMS.

\subsection{Limitations of the Experiment}

In an actual market, a participant who has used a particular handle for only a short duration could be simply be new to the market. In our experiment, however, all participants entered into the market at the same time and therefore knew that there were no new users. As a result, they might conclude that any user with an ID for any duration of ID as an indicator of trustful person. Hence, the effect of the duration should be discussed again in future experiments.

Moreover, in an actual market, the rating for the quality of a transaction is evaluated by a participant based on a subjective point of view, although here we assume that the evaluation was recorded by the RMS system based on an objective point of view, i.e. cooperate or defect. We took into account the robustness of reputation information against unfair evaluation as discussed by Dellarocas (2000). In our experiment, we ignored that the evaluation method in our current system is objective (i.e., the system just records cooperative and defective actions) rather than subjective (e.g., the seller sent me a good item quickly). In future experiments, we will investigate the effects of subjective evaluation by participants. 


\section{Summary}

We developed an experimental system to analyze the effects of a reputation management system (RMS) on promoting cooperative behavior by participants and to reveal information behavior in an online C2C market. According to the results, over $80 \%$ of participants behaved cooperatively due to the RMS. Moreover, based on analysis of information behavior, we also found that cooperative participants often referenced the number of defective actions and duration of handle. In the experiment, no new users entered the market. The situation was predicted by Yamamoto et. al. (2004) using computer simulation. The results conclude that negative RMS based on the number of defects are effective in promoting cooperative behavior when there are few new users. Based on the results, we proposed two principles for redesigning future RMS for secure online $\mathrm{C} 2 \mathrm{C}$ transaction. In future research, we will investigate online $\mathrm{C} 2 \mathrm{C}$ markets in a dynamic environment whereas participants are always changing.

\section{Notes}

1) 18 of 37 participants were always cooperative, while the others were sometime defective.

2) The result of discriminant analysis and the distribution of discriminant points.

3) The number of effective answers was 36, while there were 37 participants.

4) News report by Asahi Online, Jan. 13, 2005 (http://www.asahi.com)

\section{References}

1. Bolton, G. E., Katok, E. and Ockenfels, A. "How effective are online reputation mechanisms? An experimental study" Management Science 50(11) 1587-1602.2004.

2. Dellarocas C., "The Digitization of Word of Mouth: Promise and Challenges of Online Feedback Mechanisms", Management Science, Vol. 49, No. 10, pp.1407-1424, 2003.

3. Dellarocas, C., "Immunizing Online Reputation Reporting Systems against Unfair Ratings and Discriminatory Behavior", Proceedings of the 2nd ACM Conference on Electronic Commerce, pp.17-20, 2000.

4. Kollock, P., "The Production of Trust in Online Markets", Advances in Group Processes, Vol.16, pp.99-123, 1999.

5. McDonald, C., and C. Slawson, "Reputation in An Internet Auction Market", Economic Inquiry, vol.40, issue 4, pp.633-650, 2002.

6. Resnick, P. and Zeckhauser, R., "Trust among strangers in internet transactions: Empirical analysis of eBay's reputation system", Technical report, University of Michigan, 2001.

7. Rice, S.,"Online Reputations with Noisy Transactions: An Experimental Study", 2004 Workshop on Information Systems and Economics.

8. Ruth,J., and York,A.,"Framing information to enhance corporate reputation: The impact of message source, information type, and reference point",Journal of Business Research vol.57, pp.14-20,2004.

9. Yamamoto, H., K. Ishida and T. Ohta, "Modeling Reputation Management System on Online C2C Market", Computational \& Mathematical Organization Theory, Vol. 10, No. 2, pp.165-178, 2004. 